Revue d'histoire de l'Amérique française

RAS REVUE D.HISTOIRE DE L'AMÉRIQUE FRANÇAISE

\title{
La stratification sociale : une direction de recherche
}

\section{René Jetté}

Volume 26, numéro 1, juin 1972

URI : https://id.erudit.org/iderudit/303150ar

DOI : https://doi.org/10.7202/303150ar

Aller au sommaire du numéro

Éditeur(s)

Institut d'histoire de l'Amérique française

ISSN

0035-2357 (imprimé)

1492-1383 (numérique)

Découvrir la revue

Citer cet article

Jetté, R. (1972). La stratification sociale : une direction de recherche. Revue

d'histoire de l'Amérique française, 26(1), 33-52. https://doi.org/10.7202/303150ar d'utilisation que vous pouvez consulter en ligne.

https://apropos.erudit.org/fr/usagers/politique-dutilisation/ 


\title{
LA STRATIFICATION SOCIALE : UNE DIRECTION DE RECHERCHE *
}

\author{
RENÉ JeTtÉ \\ Département d'histoire \\ Cegep Bourgchemin \\ (Campus de Saint-Hyacinthe)
}

Le sujet de notre thèse se situait dans le cadre des recherches, tant historiques que socio-économiques, portant sur les structures sociales du Québec aux différentes étapes de leur évolution. Son objectif particulier était, essentiellement, de proposer, à titre d'hypothèse de travail, une méthode d'approche permettant de donner une sorte de définition descriptive des différents niveaux des structures sociales de la Nouvelle-France.

On se limitera à exposer ici l'hypothèse de travail elle-même, quitte à l'illustrer au passage par des données tirées de son application au régime français. D'abord, parce que le cadre restreint d'une thèse de maîtrise ne permettait pas d'en utiliser à fond toutes les potentialités, de sorte que les conclusions que l'on pourrait en tirer à propos des classes sociales en NouvelleFrance seraient plus indicatives que probantes. Mais surtout, il faut avouer que le cadre historique choisi n'était, à la limite, qu'un prétexte pour illustrer une méthode d'approche du problème de la stratification sociale qui reste applicable à bien d'autres cadres spacio-temporels.

A ceux qui s'insurgeraient contre l'introduction en histoire de modèles théoriques, qu'il suffise de faire remarquer que toute science ne fonctionne pas autrement que par un constant aller-retour entre les faits et les hypothèses permettant de les classer, de les comprendre et de les expliquer. Il est temps, par contre, que l'on se fasse à l'idée que "la sociologie est due à une conception trop étroite de l'histoire", et que "l'articulation ac-

* La direction de recherche dont l'exposé fait l'objet des pages suivantes a été appliquée à la société canadienne sous le régime français dans une thèse de Maîtrise ès Arts, thèse présentée par l'auteur à la Faculté des Lettres de l'Université de Montréal en 1970 sous le titre : Les structures sociales de la Nouvelle-France : une direction de recherche.

[33 ]

RHAF, vol. 26, no 1 (juin 1972) 
tuelle des disciplines ... est calquée en gros sur la différence des documentations", de sorte qu' "il n'est pas nécessaire de prendre la différence des documentations pour une différence épistémologique". 1

\section{POSITION DU PROBLÈME}

On pourrait, pour fixer les idées, résumer de la façon suivante les différentes phases des recherches portant sur la stratification sociale : 1 - description, puis explication de la stratification (strates, ou groupes sociaux, de même que leur hiérarchie) ; 2 - description, puis explication de la mobilité sociale; 3 - description, puis explication de l'évolution, et de la stratification, et de la mobilité.

La direction de recherche qui sera proposée au cours de cet article se situe fondamentalement au niveau de la description, aussi précise et nuancée que possible, des groupes sociaux, de leur organisation hiérarchique et de la forme et du degré de mobilité qu'ils autorisent, d'une part, et de leurs évolutions respectives, d'autre part. Il s'agit donc simplement d'une méthode de classification qui ne fait que présenter dans un ordre rationnel et justifié un ensemble de faits auxquels elle reste toujours intimement reliée.

Non que des définitions descriptives et des classifications ne puissent suggérer des pistes de recherche au niveau de l'explication, mais la préoccupation essentielle qui a orienté les recherches et la réflexion ayant abouti à la direction de recherche proposée ici était de répondre à cette question: comment décrire la stratification d'une société sans faire appel à des définitions ou à des critères qui y opéreraient un découpage artificiel ou partiel, sinon partial ?

On objectera (avec raison, espérons-le !) que l'époque est révolue où historiens comme sociologues plaquaient systématiquement sur des groupes humains, avec les mots, les définitions empruntées à des sociétés différentes dans le temps ou l'espace, voire même à quelque doctrine légale ou philosophique. C'est un fait qu'on utilise avec plus de circonspection les étiquettes de "noblesse" ou de "bourgeoisie", mais à force de faire varier

1 Paul Veyne, Comment on écrit l'histoire. Essai d'épistémologie (Paris, Seuil, coll. "L'Univers historique", 1971), 332-333. - Le lecteur pourra, pour toute cette discussion sur la nature de l'histoire qui n'a pas sa place dans ces lignes, se référer fort utilement à cet ouvrage qui, soit dit en passant, n'est pas dépourvu d'un humour pour le moins inattendu dans un volume de ce type... 
leur contenu pour l'adapter à la réalité de chaque société, on risque, à l'inverse, de ne plus rien décrire de spécifique.

Il faut cependant reconnaître qu'on a aussi abordé l'étude des structures sociales sous un angle, sinon plus empirique ${ }^{2}$, du moins plus rassurant par la précision et les limites imposées par les critères utilisés eux-mêmes. C'est d'ailleurs par le biais de cette méthode d'approche que sera introduite maintenant la direction de recherche proposée. Car celle-ci repose, globalement, sur l'utilisation conjointe de deux des critères généralement reconnus comme révélateurs de la structuration sociale : la fonction et le mariage; d'où le nom d' "endogamie fonctionnelle" donné au critère double qui en constitue la base.

\section{LE CHOIX DES CRITERES}

Mais pourquoi, parmi tous les critères permettant de répartir les individus à l'intérieur d'une hiérarchie sociale quelconque, n'avoir conservé que deux critères seulement, et ceux-là en particulier ? Car même en reconnaissant qu'il ne peut être question, en pratique, de combiner tous les facteurs imaginables afin d'obtenir, grâce à leurs recoupements, l'image la plus réaliste possible des structures sociales analysées, on peut se demander à juste titre pourquoi privilégier les seuls critères de la fonction et du mariage. Et dans ce cas, pour quelles raisons les employer conjointement plutôt que successivement?

On peut s'interroger, tout d'abord, sur la valeur réelle, même au plan théorique, du moyen mécanique qui consisterait, en sorte, à additionner les résultats obtenus par chacun des " $\mathrm{x}$ " critères retenus. La somme des éclairages particuliers que forment les hiérarchies propres à chaque critère provoque, bien sûr, des recoupements, des concentrations significatives, mais elle multiplie aussi d'autant les exceptions, elles-mêmes réparties dans des directions divergentes, au point de compromettre, tant par leur nombre que par leur dispersion, l'énoncé de toute description cohérente, et, a fortiori, de toute explication.

Par ailleurs, la constatation de l'existence de plusieurs typologies, jointe à l'observation des faits la plus élémentaire, démontre que tous les critères utilisables n'ont pas la même importance, la même valeur, que certains sont subordonnés à d'autres,

2 Ce qui, faut-il préciser, est de toute manière aussi illusoire qu'ascientifique. Parcourir, notamment: Jean Piaget, dir., Logique et connaissance scientifique (Paris, Gallimard, "Encyclopédie de la Pléiade", 1967), 1345 pages. 
et qu'en définitive, il importe plutôt d'identifier les facteurs fondamentaux, les plus révélateurs, bien que les autres permettent un enrichissement au tableau d'ensemble dans le sens de la précision, de la nuance, ou, tout simplement, de la confirmation de la classification qu'il supporte.

Il serait honnête de signaler aussi, puisque la présente hypothèse de travail laisse de côté cet aspect du problème, comme d'ailleurs pratiquement toute la littérature sur le sujet, qu'il faudra un jour tenir compte de la possibilité que les facteurs de stratification comme de mobilité sociales varient dans le temps et l'espace, fût-ce à des degrés divers, et qu'en conséquence, l'importance relative des critères qui apparaissent les plus fondamentaux dans n'importe quelle société peut évoluer, elle aussi. ${ }^{3}$

Il ne saurait donc être question de passer en revue tous les critères utilisés ou utilisables, ni même de discuter in extenso ceux qui ont été retenus à la lumière des données de la sociologie ${ }^{4}$, mais plutôt de condenser l'essentiel des acquisitions faites à la suite des diverses analyses portant aussi bien sur le "présent" que sur le "passé". Or, trois critères se détachent nettement de l'ensemble comme particulièrement révélateurs du niveau ou du statut social d'un individu: la richesse, la fonction et le mariage. ${ }^{5}$

${ }^{3}$ Il est bien possible, par exemple, que le mariage (pour utiliser le critère pivot de la présente direction de recherche...) ait d'autant moins de signification sociale que sa rupture devienne (ou ait été) aussi aisée que normale, quelles qu'en soient les raisons. Entre les Etats allemands légiférant sur le mariage morganatique et les communes "hippies", le rôle du mariage comme indicateur de la structuration sociale varie inévitablement beaucoup en importance.

4 Consulter, entre autres : Harry M. Johnson, Sociology : A Systematical Introduction (Harcourt, Brace and World Inc., New York, 1960), 688 pages. - Bernard Barber, Social Stratification. A Comparative Analysis of Structure and Process (Harcourt, Brace and World Inc., New York, 1957), 540 pages. - John Porter, The Vertical Mosaic (University of Toronto Press, Toronto, 1965), 626 pages. - Roland Mousnier, Les hiérarchies sociales de $1450 \grave{a}$ nos jours (P.U.F., Paris, coll. "sup", section "L'historien", 1969), 196 pages.

5 Il est d'une importance capitale de préciser, à ce moment-ci, que les justifications d'allure sociologique données à ces critères ne sont pas, en réalité, l'unique base de l'hypothèse de travail présentée au cours de cet article. Car non seulement le recours aux ouvrages sociologiques a été précédé d'une longue enquête documentaire, dont les renseignements ont entraîné l'auteur à élaborer lui-même les premières hypothèses de travail, mais les conclusions des ouvrages sociologiques ont été elles-mêmes, sinon systématiquement vérifiées, du moins confirmées par la documentation accumulée, laquelle se résume, en fin de compte, à un ensemble de fiches portant des noms d'individus, des fonctions, des mariages... 


\section{La richesse}

La richesse (fortune, revenu, etc.) d'un individu est sûrement le facteur le plus séduisant à utiliser. Car même si elle n'est pas toujours facile à établir, pour des raisons d'ordre documentaire surtout, elle se prête aisément à une classification hiérarchisée et précise des individus. Ce n'est pas par hasard, si, pour s'en tenir à l'histoire de la Nouvelle-France, Frégault, Hamelin et Nish utilisent des critères d'ordre économique pour évaluer le niveau social de la bourgeoisie canadienne. ${ }^{6}$ Il s'agit là pourtant d'un critère dangereux et dont la valeur devient même fort discutable lorsqu'il est utilisé seul.

Il suffit d'abord de signaler qu'un chiffre n'a de sens qu'en relation avec d'autres, de sorte qu'une échelle sociale fondée sur la richesse doit s'appuyer sur des points de comparaison. Mais alors que ce problème peut facilement être écarté, pourvu qu'on en prenne conscience ? , il devient plus complexe d'établir le statut social d'un individu pour l'ensemble de sa vie en ne considérant que sa fortune, puisque, dans bien des cas, elle peut varier considérablement sans que cela implique une variation parallèle sensible de son statut. L'un des plus grands marchands de la Nouvelle-France, Charles Aubert de la Chesnaye, est mort cousu de dettes, mais ses descendants n'en ont pas moins gardé leur rang (entre autres, probablement, à cause de la noblesse accordée à sa famille).$^{8}$

Mais même dans les cas où cette seconde difficulté peut se résoudre avec une précision satisfaisante (en considérant l'ensemble de la carrière du personnage plutôt que sa fortune à un moment précis), l'utilisation de ce critère reste entachée d'une faiblesse fondamentale, du fait que le niveau de la fortune ou du revenu d'un individu ne correspond pas nécessairement à son statut social. A revenus égaux, un marchand ou un avocat, ou un policier ou un professeur, n'ont pas encore un statut égal dans la société québécoise contemporaine, bien que nous vivions dans une période d'intenses transformations socio-économiques.

Le fait que ces deux échelles de valeur, richesse et statut social, tendent à se superposer à long terme n'implique pas

6 Guy Frégault, La société canadienne sous le régime français, brochure de la Société historique du Canada (Ottawa, 1960), 16 pages. - Jean Hamelin, Economie et société en Nouvelle-France (P.U.L., Québec, 1960), 137 pages. - Cameron Nish, Les bourgeois-gentilshommes de la NouvelleFrance, 1729-1748 (Fides, Montréal et Paris, 1968), 202 pages.

7 Cf. Nish, op. cit., entre autres aux pages 17-19.

8 Cf. Pierre-Georges Roy, La famille Aubert de Gaspé (Lévis, 1907), 199 pages. 
qu'elles coïncident effectivement à un moment donné; l'écart peut varier, mais le décalage ne disparaît jamais complètement.

La richesse se présente plutôt, soit comme un moyen d'accéder à un certain niveau social (le cas typique en est l'anoblissement des riches bourgeois comme les Fugger devenus comtes puis princes du Saint-Empire, les Rotschild devenus barons en France et en Angleterre, les LeMoyne devenus barons de Longueuil en Nouvelle-France, les Smith devenus barons "of Strathcona and Mount-Royal" au Canada, etc.), soit comme la conséquence d'une ascension sociale (c'est ainsi, par exemple, qu'une élévation dans une hiérarchie quelconque se manifeste normalement par une augmentation de salaire ou des sources de revenu; il est probable, de même, que le relèvement rapide et relativement récent des traitements accordés aux enseignants ait été dû en bonne partie au prestige nouveau accordé à l'éducation au Québec).

Une classification sociale fondée sur la richesse reste donc puissamment indicative, puisque les discordances restent, au total, moins nombreuses que les concordances; mais elle laisse tout de même trop d'exceptions sans explication : non pas tant parce qu'il s'agit d'un grand nombre de cas individuels, mais parce que ce sont souvent des ensembles de cas homogènes qui ne réussissent pas à entrer dans les cadres de ce type de critère.

Il serait toutefois injuste de passer sous silence une variante importante du critère "richesse", qui se rapproche aussi, par certains aspects, du critère "fonction" : la répartition des individus en classes sociales en fonction de la source de leurs revenus (propriété, capital, salaire, Etat). Il est fort possible que cette hypothèse se révèle beaucoup plus fructueuse qu'on a peut-être osé le penser, au niveau de l'explication, mais à condition d'être fondée sur une description de la stratification beaucoup moins sommaire que celle à laquelle elle donne lieu. Qui, en effet, pourrait envisager sérieusement de séparer (voire même d'opposer) le cultivateur propriétaire d'une exploitation familiale de l'ouvrier salarié, mais d'inclure celui-ci dans la même "classe" que le fonctionnaire, sinon le juge, tandis que tous ces gens ont possiblement transformé en capital une fraction plus ou moins grande de leur épargne...?

\section{La fonction}

"Most attempts to describe the class structure of any particular society place great emphasis on occupation or its equi- 
valent." 9 Les différentes fonctions (ou rôles) exercées dans la société peuvent, en effet, servir aussi de base à une hiérarchisation des classes sociales qui serait fondée sur l'importance relative de chaque fonction. Le critère permettant d'évaluer cette importance est le degré de responsabilité qu'elle implique, et par conséquent, d'influence, de pouvoir de décision, ou encore, le degré de non-participation directe à l'exécution des tâches.

S'il est assez aisé d'établir une hiérarchie de fonctions à l'intérieur d'un secteur d'activité déterminé (politique, militaire, économique, religieux, etc.), il est plus difficile d'imbriquer ces diverses hiérarchies dans l'ensemble des structures sociales. Sontelles parallèles ? Leurs sommets se confondent-ils ? Y a-t-il confusion des élites ? Ou bien certains groupes de fonctions sont-ils, globalement, plus élevés, et si oui, lesquels, et dans quelles sociétés ? 10

A côté de ces problèmes, l'objection fondamentale à ce type de classement demeure le fait qu'on y risque de glisser vers une description des structures professionnelles, alors que leur correspondance avec les structures sociales est loin d'être automatique. Il suffit de remarquer, par exemple, ce qu'a d'artificiel la distinction entre "classe ouvrière" et "classe agricole" au Québec: ces groupes ne se distinguent vraiment que sur le plan professionnel, et dès qu'on ne les aborde plus sur des sujets de nature strictement professionnelle, on les assimile l'un à l'autre, sans plus de distinction, à la masse du peuple; et d'ailleurs, les membres de ces deux "classes" se sentent-ils euxmêmes si distincts au point de vue statut social ?

Utilisée seule comme critère de classification sociale, la fonction laisse donc des secteurs inexpliqués, tandis qu'elle crée des distinctions inexistantes ou effectue des coupures mal placées. C'est suffisant pour en rendre l'usage suspect en dehors d'une étude portant spécifiquement sur les structures professionnelles.

\section{Le mariage}

Le mariage appartient à la catégorie des critères appelés "attitudes" ou "comportements". Or, à cause de leur caractère subjectif, la valeur de ceux-ci peut varier considérablement d'un

9 Johnson, op. cit., 485.

10 Tels sont les problèmes que pose, entre autres, l'ouvrage de Porter, de même, d'ailleurs, que celui de Mousnier, sous un angle différent. 
critère à l'autre, d'une part, et d'autre part, elle est souvent difficile à estimer (soit qu'ils prennent une forme passagère, ou encore qu'ils puissent avoir plusieurs sens, selon les circonstances, etc.). C'est ainsi, par exemple, que des marques exceptionnelles de sympathie (assister à un mariage, accepter d'être parrain, etc.) n'impliquent pas nécessairement une reconnaissance d'égalité sociale; dans bien des cas, l'inférieur considère comme un honneur une telle marque d'intérêt à son égard, laquelle, par ailleurs, peut avoir été donnée le plus souvent comme une faveur, ou, plus rarement, par amitié.

Les sociologues s'accordent toutefois à reconnaître que "marriage is the most complete and reliable expression of social intimacy and social equality ... Except for marriage perhaps, no single kind of behavior can be reliably taken as expressive of social equality" ${ }^{11}$; "the most decisive mark of class equality between families is the fact that they will accept one another's children in marriage without feeling, on either side, that the match was socially inappropriate". ${ }^{12}$ C'est sur la foi de ces témoignages ${ }^{13}$ que la seule attitude considérée comme critère suffisamment valable de distinction sociale au cours de cet exposé est le mariage, ou, plus précisément, le taux d'endogamie.

On pourrait définir ce dernier comme suit: le rapport entre le nombre de mariages contractés à l'intérieur de leur propre groupe par les individus (hommes et/ou femmes, selon le genre d'analyse) d'un groupe quelconque, d'une part, et le nombre total des mariages contractés par ceux-ci, d'autre part, compte tenu de la proportion des individus mariés dans chacun des groupes concernés. Par opposition, le taux d'exogamie correspond aux mariages contractés en dehors de leur propre groupe. Ce groupe peut aussi bien être une famille qu'une ethnie (l'endogamie ethnique a été particulièrement étudiée aux EtatsUnis) ou une classe ("class endogamy" ${ }^{14}$ ).

Une famille, une ethnie, ce sont là des groupes faciles à circonscrire, à définir, avant d'y étudier le degré d'endogamie interne. Mais ce n'est plus aussi simple lorsqu'on parle d'endogamie de classe, puisque ce sont précisément les différents niveaux des structures sociales que l'on désire délimiter grâce à ce critère.

11 Barber, op. cit., 130 et 131.

12 Johnson, op. cit., 471.

13 Relire, toutefois, la note 5.

14 Johnson, op. cit., 471. 
Il y a des cas évidents, bien sûr, et ce sont d'ailleurs ceux-là qui ont servi de base aux recherches effectuées à l'aide de ce critère. Il y a tout d'abord le cas traditionnel du prince qui doit renoncer à sa bergère pour épouser quelque princesse de son rang, ou encore, en version américaine, celui du fils du grand patron qui courtise la fille d'un des ouvriers de son père. D'autre part, une lecture même rapide de l'ancien Almanach de Gotha européen, du Burke's Peerage britannique ou du Social Register américain révèle à l'évidence un taux d'endogamie de classe particulièrement élevé entre les "grandes familles".

Pourtant, ces deux groupes d'exemples recèlent, chacun à sa manière, la faiblesse fondamentale de ce critère : pour l'utiliser, il faut accepter au point de départ une définition de classe. Le premier exemple utilise des éléments qui se rapportent plutôt au statut professionnel; mais étant donné que la concordance générale entre le statut professionnel et le statut social est trop claire dans ce cas, le facteur endogamie ne fait que la confirmer; or, ce ne sont pas les cas extrêmes qui sont les plus intéressants, les plus révélateurs. Quant au second exemple, il utilise la famille comme critère; or, non seulement le statut d'une famille est déterminé par plusieurs facteurs, mais, surtout, ce statut peut varier, en particulier au long des générations, de sorte qu'on ne peut réduire l'endogamie de classe aux alliances matrimoniales contractées entre un certain nombre de familles dont le choix doit d'abord être justifié lui-même.

Cette justification est relativement aisée pour les familles dont le nom et l'histoire sont consignés dans des ouvrages qui leur donnent une reconnaissance officielle de statut, mais le reste de la population ne forme certainement pas une classe homogène : il est évident, par exemple, que les Godefroy de Tonnancour, simples cultivateurs de la région d'Yamaska dans la seconde moitié du XIX ${ }^{\mathrm{e}}$ siècle, étaient bien éloignés du niveau social de leurs ancêtres du XVII et même du XVIII ${ }^{e}$ siècle. ${ }^{15}$ Il faudrait pourtant pouvoir mesurer cette distance sociale avec plus de précision, plus de nuances, en décrivant en particulier les étapes de l'évolution qui s'est produite entre ces deux époques.

Malgré toutes ces difficultés, l'endogamie de classe reste peut-être le critère le plus fonctionnel de tous ceux qui ont été exposés, à condition d'être complété. Son caractère subjectif le rend en effet certainement plus révélateur, dans la mesure où

${ }_{15}$ Pierre-Georges Roy, La famille Godefroy de Tonnancour (Lévis, 1904), 128 pages (passim). 
la conscience qu'ont les individus des différents niveaux sociaux est plus importante qu'une classification fondée sur des critères objectifs, extérieurs (comme le sont la richesse et la fonction) ${ }^{16}$; et surtout, ce critère sous-tend tout un système de valeurs et d'attitudes (y compris, en un sens, la richesse et la fonction) commun aux familles des époux ainsi qu'aux époux eux-mêmes, ce qui lui confère un caractère éminemment synthétique, global.

Mais comment utiliser ce critère sans définir auparavant la (ou les) classe à laquelle on désire l'appliquer ? C'est ce dilemme que l'utilisation conjointe de la fonction et de l'endogamie permet de résoudre.

\section{L'endogamie fonctionnelle}

Il faut toutefois répondre immédiatement à l'objection suivante: pour quel motif préférer adjoindre au critère endogamie le critère fonction, plutôt que le critère richesse ? Il faut reconnaître, en effet, que l'un et l'autre figurent parmi les critères les plus universellement reconnus et recommandés, malgré les faiblesses qu'entraîne l'emploi d'un seul de ces critères à la fois.

Cependant, le seul argument qui paraisse valable pour justifier cette préférence est d'ordre pratique. Les recherches sur l'histoire des structures sociales au Québec, en particulier, bénéficient d'une richesse documentaire surabondante et, par surcroît, presque inexplorée dans le sens de l'étude des structures sociales. Il apparaît donc nécessaire et souhaitable de tenter de déblayer, d'ordonner à grands traits cette mine de renseignements que constituent les archives, judiciaires et autres, au risque de généraliser trop tôt ou même de fausser le sens des faits.

Ce risque est tout de même bien calculé : les deux critères retenus sont des plus sérieux, et leur combinaison à l'intérieur de l'endogamie fonctionnelle leur confère encore plus de valeur, comme il sera démontré plus loin. Mais il ne suffit pas que les critères soient valables et que l'hypothèse de travail puisse tenir compte du plus grand nombre d'aspects possible : il s'agit en même temps de classer rapidement et significativement la documentation accessible.

16 "Pour fondamental que puisse paraître aux yeux de beaucoup le problème de l'antériorité logique, historique et psychologique des facteurs objectifs sur les facteurs subjectifs, que le lecteur soit assez indulgent pour permettre à l'auteur d'esquiver ici la démonstration de cette affirmation. Qu'il se console à la pensée que l'endogamie fonctionnelle tient également compte du critère fonction ...." 
Or, l'établissement du degré de richesse d'un individu, surtout tout au long de sa vie, requiert le recours à une somme considérable de documents, lesquels sont, en plus, très divers et relativement dispersés. Par contre, la source unique que constituent les registres d'état civil, et, à l'intérieur, les actes de mariages, peut fournir tous les renseignements nécessaires pour se servir du critère endogamie, et, dans l'ensemble, suffisamment de données sur les fonctions exercées par les individus pour rendre utilisable le critère fonction. ${ }^{17}$

En résumé, trois critères tranchent nettement sur les autres comme indicateurs du niveau social : la richesse, la fonction et le mariage. Mais utilisés seuls, ils sont entachés de défauts peu rassurants sur la valeur des conclusions que l'on peut tirer de leur application. Il apparaît toutefois que le dernier, le mariage, serait peut-être le plus révélateur de tous, si seulement il ne fallait pas d'abord définir le groupe où l'on désire analyser ce facteur; or, cette opération devient impossible dès qu'on parle d'endogamie de classe, ce dernier terme devant justement faire l'objet de l'enquête. Il devient donc nécessaire de jumeler l'endogamie à un autre critère, et, dans ces circonstances, la fonction s'avère plus commode que la richesse pour jouer ce rôle, à possibilités théoriques comparables.

En combinant les critères endogamie et fonction, il est non seulement possible de résoudre le dilemme que pose la notion d'endogamie de classe, mais aussi de supprimer en grande partie les inconvénients de l'utilisation indépendante du critère fonction, permettant ainsi d'obtenir, par le critère double d'endogamie fonctionnelle, des résultats supérieurs à n'importe lequel des critères pris séparément; c'est ce qui reste maintenant à expliquer.

Par définition, l'utilisation du taux d'endogamie implique nécessairement la présence d'au moins deux groupes distincts :

17 Dans la mesure, évidemment, où les registres d'état civil, pour le pays et l'époque étudiés, possèdent au moins les caractéristiques des registres paroissiaux catholiques du Québec : lacunes rares, d'une part, et règles d'inscription des actes aussi exigeantes. On notera ici, une fois de plus, jusqu'à quel point le caractère et la qualité de la documentation accessible peuvent non seulement faciliter, mais orienter la méthodologie comme l'objet de la recherche. Des conditions de travail moins favorables rendraient probablement compte en bonne partie du fait que les analyses utilisant le critère mariage soient restées aussi rares que superficielles. Par contre, la direction de recherche proposée ici peut aisément s'accommoder de la combinaison d'autres sources (ce qui a dû être fait même pour le régime français). 
deux familles, deux ethnies, deux classes, etc., selon le cas. Ainsi, au sens strict, l'endogamie fonctionnelle est le rapport entre le nombre de mariages contractés à l'intérieur de leur propre fonction par les titulaires d'une fonction donnée, et le nombre total des mariages contractés par ceux-ci (qu'il y ait deux ou cent fonctions, peu importe). Cette notion, grâce à sa souplesse et à sa précision, permet d'effectuer un nombre considérable de regroupements en même temps qu'elle rend possible l'utilisation des plus infimes distinctions sociales.

La fonction d'officier militaire, par exemple, peut être, d'une part, subdivisée, soit en énumérant chacun des grades possibles comme autant de fonctions distinctes, soit en lui ajoutant une ou plusieurs fonctions pour construire autant de fonctions complexes (v.g. : officier militaire et seigneur; officier militaire, seigneur et marchand; etc.) ; et d'autre part, on peut regrouper un certain nombre de fonctions et les traiter globalement comme une seule fonction (mais qu'il serait préférable de nommer "groupe de fonctions", pour éviter la confusion).

De cette manière, non seulement le dilemme dans lequel se trouve l'expression d'endogamie de classe disparaît parce que l'étiquette "classe" 18 peut être précisée dans son extension par les fonctions simples ou complexes qu'on peut y loger, mais les séparations peu ou pas significatives qui existent inévitablement entre toutes les subdivisions possibles d'une société selon les fonctions qu'on y exerce sont susceptibles de disparaître grâce aux regroupements suggérés par leurs taux d'endogamie (en même temps, par ailleurs, que ces derniers peuvent indiquer la nécessité de subdivisions nouvelles ou différentes).

L'utilisation conjointe du mariage et de la fonction va donc plus loin qu'un complément ou qu'une vérification réciproque. Il ne s'agit pas, en effet, de la comparaison des résultats de deux démarches successives, mais de l'intégration de deux variables à l'intérieur d'un critère double.

Il est bon de rappeler que dans la mesure où l'on considère que la richesse constitue un critère aussi valable que la fonction pour déterminer le statut social d'un individu, il pourrait théoriquement remplacer la fonction à côté du mariage ${ }^{19}$;

18 Faut-il préciser que partout où le terme "classe" apparaît au cours de ce texte, il n'est utilisé que pour simplifier le vocabulaire, sans préjuger aucunement de son sens? C'est une étiquette commode visant simplement à recouvrir des réalités aussi vagues que des distinctions de niveaux à l'intérieur d'une hiérarchie sociale.

19 Ce qui devrait toutefois être discuté et démontré. 
mais il ne faut pas réfléchir longtemps pour en abandonner l'idée devant la difficulté, sinon l'impossibilité plus ou moins fréquente, d'établir le degré de fortune d'un individu à un moment donné. Mais cela n'empêche pas que ce critère puisse apporter des précisions utiles, indispensables même dans certains cas. Ainsi, par exemple, il peut arriver qu'un riche marchand d'humble origine se soit marié avant d'avoir atteint le degré de richesse qui lui a permis de tenir un rang élevé dans la société et de "bien" marier ses enfants (c'est le cas, entre autres, de Charles LeMoyne et de Joseph Cadet, en Nouvelle-France). De sorte que ces exceptions du point de vue endogamie fonctionnelle peuvent être expliquées en faisant l'histoire de leur carrière sur le plan économique.

\section{LE FONCTIONNEMENT DE LA MÉTHODE}

La méthode de description et de classification des structures d'une société proposée dans cet article repose donc, en définitive, sur un critère unique, mais double, appelé endogamie fonctionnelle. Il en découle un certain nombre de principes de base qu'il reste maintenant à exposer, de même qu'à décrire le mécanisme général permettant de l'appliquer à des situations concrètes, tout en en faisant ressortir à la fois la portée et les limites.

\section{Principes de base}

Les principes de base énoncés plus bas constituent en quelque sorte une série de définitions et de postulats découlant directement de la notion d'endogamie fonctionnelle; à ce titre, ils seront considérés comme suffisamment évidents pour ne pas devoir être démontrés.

1 - Le terme "fonction" est employé dans un sens élargi et neutre pour désigner toute distinction spécifiant le rôle joué ou la place tenue par un individu dans la société. Un métier ou une profession (notaire, menuisier, marchand, cultivateur, etc), un poste ou un emploi civil ou militaire quelconque (juge, gouverneur, officier, etc.), toute marque d'un statut particulier (titre de noblesse, distinction honorifique, possession d'une seigneurie, etc.) sont considérés comme des fonctions. Celles de Charles Aubert de la Chesnaye, par exemple, seraient énumérées ainsi : noble, marchand, seigneur, membre du Conseil Souverain, etc. 
2 - Une société sera dite sans classes si le taux d'endogamie existant à l'intérieur d'une classe donnée est égal à la proportion que les membres de cette classe représentent par rapport à la population totale. Ainsi, une société où les marchands compteraient pour $5 \%$ de la population devrait alors trouver dans cette classe un taux d'endogamie de $5 \%$, correspondant à l'attente statistique. C'est parce qu'il n'est jamais réalisé en pratique que ce postulat constitue le fondement même d'une analyse utilisant le critère mariage.

3 - Si deux classes ont un taux égal d'endogamie, celle qui compte la plus faible proportion de personnes par rapport à l'ensemble de la population est considérée comme la plus élevée dans l'échelle sociale. Par exemple, un taux d'endogamie de $90 \%$ n'a pas le même sens chez les cultivateurs (qui peuvent compter pour $80 \%$ de la population) et chez les nobles (qui ne représentent peut-être même pas $1 \%$ de celle-ci).

4 - Si deux classes ont la même proportion de personnes par rapport à la population totale, celle où le taux d'endogamie est le plus élevé est considérée comme la plus élevée dans l'échelle sociale. Si, par exemple, il y a autant de familles nobles que de familles de marchands, mais que le taux d'endogamie du premier groupe est de $90 \%$ et celui du second de $50 \%$, la classe des nobles sera considérée comme supérieure à celle des marchands. Et comme ce principe ne concerne que la cohésion interne du groupe, il vaudrait même si tous les autres nobles avaient épousé des paysannes, et les autres marchands des nobles.

5 - Les classes entre lesquelles les mariages se contractent indistinctement sont considérées comme faisant partie de la même couche sociale. Ainsi, si les notaires épousent autant de filles de médecin que les médecins des filles de notaire, ces deux fonctions seront considérées comme égales.

6 - Enfin, plus les taux d'endogamie sont élevés (dans l'ensemble de la société ou entre certaines classes seulement), plus les structures sociales sont considérées comme distantes, fermées. Le cas le plus souvent cité d'une société "fermée" est celui de l'Inde (castes), tandis que le cas américain est représentatif des sociétés dites "ouvertes".

Bien entendu, l'application de ces principes suppose que toutes les autres conditions sont identiques, ce qui est loin de la réalité, et il est nécessaire de faire abstraction, éventuellement 
par des procédés statistiques, de certains éléments perturbateurs. L'endogamie fonctionnelle peut être orientée, par exemple, par l'endogamie géographique, ethnique ou religieuse: ce sont là des phénomènes intéressants à étudier en eux-mêmes, mais qu'il faut pouvoir isoler; c'est ainsi que, sous le régime français, on peut être amené à distinguer une aristocratie québécoise, une aristocratie montréalaise et même une aristocratie de la région trifluvienne.

\section{Etapes $d u$ fonctionnement}

L'application des principes de base qui viennent d'être énoncés doit évidemment être mise en rapport avec les faits, et c'est à cette fin qu'il convient maintenant d'exposer, dans ses lignes générales, les étapes du fonctionnement de la méthode d'approche fondée sur l'endogamie fonctionnelle.

Une fois déterminé le cadre chronologique et géographique où l'on désire étudier la stratification sociale, il s'agit de relever systématiquement tous les mariages contractés à l'intérieur de la période et du territoire désignés, afin d'en tirer les renseignements suivants : noms des époux et de leurs parents, fonctions de l'époux et des pères des conjoints (la profession des épouses est rarement mentionnée avant la fin du XIXe siècle dans les registres d'état civil catholiques du Québec), et, facultativement, la date et le lieu du mariage. Si le texte de l'acte de mariage contient toujours, dans les registres catholiques, les renseignements concernant le cadre familial, il est souvent incomplet avant le XIXe siècle en ce qui regarde les fonctions, de sorte qu'il peut devenir utile sinon nécessaire de le compléter par d'autres sources: actes notariés, archives officielles de tout genre, recensements, etc. Il existe toutefois certaines listes de fonctions, contemporaines ou postérieures, qui permettent d'abréger considérablement l'identification des individus un tant soit peu célèbres, en plus des dictionnaires biographiques, des Who's Who, des histoires de familles, etc.

L'étape suivante est la classification de ces données sous forme de tableaux chiffrés (pas nécessairement statistiques). Les principes de base exposés à la section précédente ont non seulement pour but de permettre leur compréhension, mais aussi de guider leur construction même. Car le nombre et la forme des tableaux peuvent varier beaucoup, selon les aspects que l'on désire mettre en relief, car ce type de documentation, pourtant relativement simple et limité, est aussi souple que polyvalent. 
Il y a toutefois un tableau fondamental dont découlent les autres, d'une façon plus ou moins directe, celui qui met en relation la fonction de l'époux et celle du père de l'épouse. C'est le seul type de tableau qui ait été utilisé dans la thèse citée au début de cet article, mais on peut imaginer l'intérêt de tableaux comme les suivants : fonction du père - fonction des fils ; fonction du père - fonction des gendres; fonction des parents des conjoints; fonction de chacun des membres d'une famille quelconque et de leurs conjoints; etc. Le choix des tableaux dépend, en fin de compte, étroitement de ce que l'on désire faire ressortir; c'est particulièrement clair dans le cas suivant.

Il n'a encore été question, en effet, que de l'analyse des structures sociales, en les considérant en dehors de leur évolution, du temps au cours duquel elles se modifient. Or, cette même méthode, par des procédés plus complexes, pourrait permettre d'étudier l'évolution même de ces structures. De plus, on pourrait construire, de la même manière, des tableaux décrivant le degré de mobilité sociale existant à un moment donné (la mobilité "inter-générationnelle", surtout) : on pourrait ainsi calculer, par exemple, quelles étaient les possibilités statistiques des enfants d'un cultivateur vivant en 1700 de devenir tous cultivateurs à leur tour, ou qu'un (deux, trois, ...) ne le devienne pas. Il serait enfin possible d'évaluer l'évolution même du degré de mobilité sociale entre des époques différentes.

Ces perspectives permettent d'entrevoir les multiples virtualités de cette direction de recherche: sa simplicité et sa souplesse s'accommodent d'une documentation singulièrement limitée en étendue et facile à rassembler, en comparaison des nombreuses possibilités qu'elle offre. Les conclusions qu'elle autorise sont à la fois suffisamment précises et nuancées pour jouer, au minimum, le rôle de déblaiement qu'elle peut entreprendre aisément.

C'est ce que l'illustration qui suit en guise de conclusion espère laisser entrevoir au lecteur.

\section{ILLUSTRATION}

Les paragraphes qui viennent présenteront, à titre d'illustration, une vue d'ensemble du découpage qu'entraîne l'endogamie fonctionnelle appliquée à l'analyse des structures sociales de la Nouvelle-France. Il importe, par conséquent, de se rendre compte qu'il ne peut s'agir que d'un aperçu global du type de 
fonctionnement de la direction de recherche exposée au cours de cet article. Une présentation plus systématique d'un exemple limité aurait comporté, par contre, deux inconvénients majeurs: la difficulté d'en bien comprendre la signification et la valeur en dehors du contexte d'une démonstration plus vaste, et surtout, l'obligation de citer, auparavant, des pages entières de données et de conventions techniques qui ne peuvent entrer décemment dans le cadre d'un article.

Il faut souligner, en commençant, que la méthode de travail n'a été appliquée systématiquement que pour tenter de définir les différents niveaux de ce qu'on peut appeler, sans préjuger du sens et de la portée de l'expression, la "classe supérieure" de la Nouvelle-France. Mais comment effectuer ainsi une coupure qui peut paraître, à bon droit, artificielle, arbitraire surtout ? Comment, en effet, délimiter ainsi à l'avance la frontière entre cette classe et le reste de la population ? Si la raison première était d'ordre pratique (nature et dimensions de la documentation), il est essentiel de se rendre compte, toutefois, que le partage préalable entre les classes "supérieure" et "inférieure" a été réalisé non seulement avec beaucoup de scrupules, mais selon les postulats même de l'endogamie fonctionnelle.

Quelles fonctions doit-on alors retenir comme susceptibles de faire partie de la classe supérieure ? Tout d'abord, il n'est pas déraisonnable de prendre en considération le fait que la Nouvelle-France était une société coloniale française des XVIIe et XVIIIe siècles, à condition, cependant, de ne pas considérer la liste des fonctions qu'on peut en inférer comme définitive et fermée. Dans ce contexte, il est permis d'éliminer de la classe supérieure les cultivateurs, les artisans, les simples soldats, et, en gros, tous ceux dont l'occupation peut être rangée dans les secteurs d'activité primaire et secondaire, et à condition, toujours, qu'il s'agisse là de leur fonction unique, naturellement. Il est évident, par contre, que les nobles, les marchands, les seigneurs, les divers officiers civils, militaires et de justice, et, en général, tous ceux dont la fonction peut être classée dans les activités tertiaires, peuvent être retenus a priori comme membres de cette classe supérieure. ${ }^{20}$

${ }^{20}$ Sur le sens des qualificatifs "primaire", "secondaire" et "tertiaire", on peut consulter, en particulier, Jean Fourastié, Le grand espoir du XXe siècle (Paris, Gallimard, coll. "Idées", no 20, 1963), 372 pages. - Cette classification socio-économique est à mettre en parallèle avec la classification d'inspiration marxiste basée sur la source des revenus et à laquelle il a été fait allusion plus haut. 
Mais pour plus de précautions, on y a ajouté diverses fonctions de type secondaire, plus proches à l'époque, du métier spécialisé, mais dont le caractère artisanal est dépassé par leur caractère artistique: telles sont les fonctions de médecin, de chirurgien, de sculpteur, d'orfèvre, d'architecte, d'armurier. De plus, une fonction hypothétique a été inventée et incluse de droit dans la classe supérieure afin de tenir compte de l'incertitude entourant certains cas particuliers: un certain nombre d'actes de mariage, en effet, citent des individus dont la fonction n'a pu être établie, alors qu'ils possédaient visiblement un statut social supérieur, soit d'après celui de leur père ou de leur beaupère, soit d'après le rang du mariage de leurs filles.

C'est donc ainsi qu'ont été isolés, avec un risque d'erreur fortement réduit, les fonctions et individus susceptibles d'appartenir à des couches de la société situées au-dessus de la classe populaire. Peut-être trop généreusement, même, car les fonctions attribuées au départ à la classe supérieure de la Nouvelle-France ne sont certainement pas toutes du même niveau social: elles s'étendent des plus hautes fonctions administratives aux fonctions les plus localisées et les plus proches du peuple; on se doute bien, par exemple, que l'huissier du Conseil Souverain et le capitaine de milice de Deschambault ne sont pas du même rang que le gouverneur de Montréal.

Mais avant de tenter une hiérarchisation à l'intérieur de cet ensemble de fonctions diverses, il est commode de conserver cette division en deux classes, afin de regarder si, à ce niveau au moins, les principes de base de l'endogamie de classe se vérifient, s'ils révèlent l'existence, à travers les mariages, d'une certaine étanchéité entre ces deux groupes sociaux numériquement très inégaux.

En admettant que la classe supérieure contienne $10 \%$ de la population du pays, on devrait y trouver, en fonction du postulat fondamental de l'endogamie, un taux d'exogamie de $90 \%$; or, il n'est que de $20.9 \%$, puisque seulement 579 des 2774 mariages recensés ont été contractés avec des filles de simples habitants. On pourrait donc affirmer dès maintenant que la Nouvelle-France a connu à un degré relativement élevé les distinctions de niveau social, et qu'à tout le moins entre ces deux groupes, il existait une distance sociale assez forte et perçue comme telle.

Par contre, un taux d'exogamie tel que le cinquième de la classe supérieure consente à s'allier aux gens du peuple reste 
suffisamment fort pour qu'on se demande pourquoi il en était ainsi. Cependant, avant d'en conclure que la société canadienne était alors une société ouverte où les classes étaient bien plus rapprochées et perméables que dans la mère patrie, il convient de distinguer différents niveaux à l'intérieur même de ce qui a été considéré jusqu'ici comme une classe homogène.

Car elle est loin de l'être, puisque les taux d'exogamie varient, selon les fonctions, entre $0 \%$ et $71 \%$, autour de la moyenne de $20.9 \%$. Le regroupement de ces taux permet de rassembler les quatorze fonctions principales ${ }^{21}$ en trois groupes bien distincts qui constitueraient autant de paliers à l'intérieur de cette classe supérieure.

Le premier groupe est formé des cinq fonctions principales dont les titulaires ont contracté moins de $10 \%$ de mariages à l'extérieur de cette classe : il s'agit des officiers militaires $(4.3 \%)$, des officiers de justice royaux $(3.8 \%)$, des officiers civils, catégorie "A" $(0 \%)$, des membres du Conseil Souverain (1.3\%) et des nobles $(10.8 \%)$. La moyenne du groupe est de $7.5 \%$.

Le second groupe est composé des trois fonctions dont le taux d'exogamie oscille autour de $20 \%$ (la moyenne du groupe étant d'ailleurs de $20.5 \%$ ) : ce sont les officiers civils, catégorie "B" $(29.6 \%)$, les marchands $(21.7 \%)$ et les seigneurs $(18.3 \%)$.

Quant au dernier groupe, il rassemble les six autres fonctions dont les taux d'exogamie, notablement plus élevés, dépassent

21 Une fonction principale est une fonction qui donne naissance à une division majeure des données statistiques. D'une part, elle recouvre un certain nombre de fonctions simples qui ont été rassemblées sous un seul chef pour réduire à une quantité maniable une cinquantaine, au moins, de fonctions simples; ainsi, par exemple, la fonction principale "justice royale" inclut les juges royaux, les procureurs généraux au Conseil Souverain, les procureurs du roi et les prévôts de la maréchaussée; de même, les officiers civils autres que ceux qui ont fait l'objet de subdivisions particulières ont été regroupés en distinguant deux catégories selon l'étendue des responsabilités : ceux de la catégorie " $A$ " ayant un mandat couvrant tout le pays (v.g. : trésorier de la Marine), ceux de la catégorie " $B$ " ayant un rôle limité à certains lieux ou à certains personnages (v.g. : écrivains).

D'autre part, une fonction principale se décompose, au cours de l'analyse, en fonctions dérivées, lesquelles sont construites par l'addition à une fonction principale d'une ou plusieurs fonctions simples (v.g. : marchand, marchand et seigneur, marchand et officier militaire, marchand et - autres fonctions -, etc.). Bien que ces regroupements et subdivisions aient forcément un caractère contestable sur le plan théorique, il n'en demeure pas moins qu'ils sont le fruit de l'observation autant que du raisonnement, et que, comme il se doit, ils sont précisés, expliqués et justifiés dans la thèse d'où ces exemples ont été extraits. 
presque tous $50 \%$ : les capitaines de milice $(71 \%)$, les médecins et chirurgiens $(64.9 \%)$, les notaires et greffiers $(55.9 \%)$, les arpenteurs $(62.5 \%)$, les artisans spécialisés $(48.6 \%)$ et les officiers de justice seigneuriaux $(60 \%)$. Ce qui donne au groupe une moyenne de $60.4 \%$.

En considérant provisoirement ces trois groupes comme autant de classes homogènes, on serait donc amené à la conclusion que les structures sociales de la Nouvelle-France étaient composées de quatre strates : celle correspondant au premier groupe décrivant, au sens strict, la véritable "classe supérieure" ou aristocratie, tandis que les groupes II et III décriraient respectivement la "classe moyenne supérieure" et la "classe moyenne inférieure", cette dernière se situant par conséquent juste audessus de la "classe inférieure", de la masse du peuple.

Mais le taux d'exogamie est loin d'être le seul facteur permettant d'évaluer le statut d'une fonction ou d'un groupe, même s'il est suffisamment indicatif pour servir de base de départ à une analyse. L'homogénéité de ces groupes et de chacune des fonctions qui les composent, leur degré de cohésion interne, la manière dont se répartissent les mariages endogames à l'intérieur de chaque groupe et aussi de chaque fonction principale, voilà autant de points à éclaircir avant d'essayer de préciser quelque définition descriptive convenable. C'est particulièrement nécessaire et significatif pour les fonctions de marchand et de seigneur, les plus hétérogènes de toutes puisque qu'une analyse approfondie permet de répartir leurs titulaires à presque tous les échelons de la hiérarchie.

Cette démonstration sort toutefois du cadre de cette illustration sommaire, les lignes qui précèdent n'ayant d'autre intention que de montrer comment l'endogamie fonctionnelle permet d'aborder l'analyse descriptive des structures sociales. Qu'elles suscitent la réflexion, la recherche et la critique, ou qu'elles provoquent des tentatives d'application, et elles auront atteint leur but. 